\title{
Bi-Immunity Separates Strong NP-Completeness Notions
}

\author{
A. Pavan ${ }^{\star 1}$ and Alan L Selman ${ }^{2}$ \\ 1 NEC Research Institute, 4 Independence way, Princeton, NJ 08540. \\ apavan@research.nj.nec.com \\ 2 Department of Computer Science and Engineering, University at Buffalo, Buffalo, \\ NY 14260. selman@cse.buffalo.edu
}

\begin{abstract}
We prove that if for some $\epsilon>0$, NP contains a set that is $\operatorname{DTIME}\left(2^{n^{\epsilon}}\right)$-bi-immune, then NP contains a set that is 2-Turing complete for NP (hence 3-truth-table complete) but not 1-truth-table complete for NP. Thus this hypothesis implies a strong separation of completeness notions for NP. Lutz and Mayordomo [LM96] and Ambos-Spies and Bentzien [ASB00] previously obtained the same consequence using strong hypotheses involving resource-bounded measure and/or category theory. Our hypothesis is weaker and involves no assumptions about stochastic properties of NP.
\end{abstract}

\section{Introduction}

We obtain a strong separation of polynomial-time completeness notions under the hypothesis that for some $\epsilon>0$, NP contains a set that is $\operatorname{DTIME}\left(2^{n^{\epsilon}}\right)$-biimmune. We prove under this hypothesis that NP contains a set that is $\leq_{2-T^{-}}^{\mathrm{P}}$ complete (hence $\leq_{3-t t}^{\mathrm{P}}$-complete) for NP but not $\leq_{1-t t}^{\mathrm{P}}$-complete for NP. In addition, we prove that if for some $\epsilon>0, \mathrm{NP} \cap c o-\mathrm{NP}$ contains a set that is

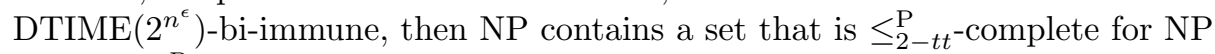
but not $\leq_{1-t t}^{\mathrm{P}}$-complete for NP. (We review common notation for polynomialtime reducibilities in the next section.)

The question of whether various completeness notions for NP are distinct has a very long history [LLS75], and has always been of interest because of the surprising phenomenon that no natural NP-complete problem has ever been discovered that requires anything other than many-one reducibility for proving its completeness. This is in contrast to the situation for NP-hard problems. There exist natural, combinatorial problems that are hard for NP using Turing reductions that have not been shown to be hard using nonadaptive reductions [JK76]. The common belief is that NP-hardness requires Turing reductions, and this intuition is confirmed by the well-known result that if $\mathrm{P} \neq \mathrm{NP}$, then there are sets that are hard for NP using Turing reductions that are not hard for NP using many-one reductions [SG77].

\footnotetext{
* Work done while the author was at University at Buffalo.
} 
There have been few results comparing reducibilities within NP, and we have known very little concerning various notions of NP-completeness. The first result to distinguish reducibilities within NP is an observation of Wilson in one of Selman's papers on p-selective sets [Sel82]. It is a corollary of results there that if $\mathrm{NE} \cap c o-\mathrm{NE} \neq \mathrm{E}$, then there exist sets $A$ and $B$ belonging to NP such that $A \leq_{\text {postt }}^{\mathrm{P}} B, B \leq_{t t}^{\mathrm{P}} A$, and $B \leq_{\text {post }}^{\mathrm{P}} A$, where $\leq_{\text {postt }}^{\mathrm{P}}$ denotes positive truthtable reducibility. Regarding completeness, Longpré and Young [LY90] proved that there are $\leq_{m}^{\mathrm{P}}$-complete sets for $\mathrm{NP}$ for which $\leq_{T}^{\mathrm{P}}$-reductions to these sets are faster, but they did not prove that the completeness notions differ. Lutz and Mayordomo [LM96] were the first to give technical evidence that $\leq_{T}^{\mathrm{P}}$ and $\leq_{m}^{\mathrm{P}}$-completeness for NP differ. They proved that if the $p$-measure of NP is not zero, then there exists a $\leq_{2-T}^{\mathrm{P}}$-complete language for NP that is not $\leq_{m}^{\mathrm{P}}$ complete. Ambos-Spies and Bentzien [ASB00] extended this result significantly. They used an hypothesis of resource-bounded category theory that asserts that "NP has a p-generic language," which is weaker than the hypothesis of Lutz and Mayordomo, to separate nearly all NP-completeness notions for the bounded truth-table reducibilities, including the consequence obtained by Lutz and Mayordomo.

Here we prove that the consequence of Lutz and Mayordomo follows from the hypothesis that NP contains a $\operatorname{DTIME}\left(2^{n^{\epsilon}}\right)$-bi-immune language. This hypothesis is weaker than the genericity hypothesis in the sense that the genericity hypothesis implies the existence of a $2^{n^{\epsilon}}$-bi-immune language in NP. Indeed, there exists a DTIME $\left(2^{n^{\epsilon}}\right)$-bi-immune language, in EXP, that is not $p$-generic [PS01]. Notably, our hypothesis, unlike either the measure or genericity hypotheses, involves no stochastic assumptions about NP.

Pavan and Selman [PS01] proved that if for some $\epsilon>0, \mathrm{NP} \cap$ co-NP contains a set that is $\operatorname{DTIME}\left(2^{n^{\epsilon}}\right)$-bi-immune, then there exists a $\leq_{T}^{\mathrm{P}}$-complete set for $\mathrm{NP}$ that is not $\leq_{m}^{\mathrm{P}}$-complete. The results that we present here are significantly sharper. Also, they introduced an Hypothesis $\mathrm{H}$ from which it follows that there exists a $\leq_{T}^{\mathrm{P}}$-complete set for $\mathrm{NP}$ that is not $\leq_{t t}^{\mathrm{P}}$-complete. We do not need to state this hypothesis here. Suffice it to say that if for some $\epsilon>0$, UP $\cap$ co-UP contains a DTIME $\left(2^{n^{\epsilon}}\right)$-bi-immune set, then Hypothesis $\mathrm{H}$ is true. Thus, we may partially summarize the results of the two papers as follows:

1. If for some $\epsilon>0, \mathrm{NP}$ contains a $\operatorname{DTIME}\left(2^{n^{\epsilon}}\right)$-bi-immune set, then NP contains a set that is $\leq_{2-T}^{\mathrm{P}}$-complete (hence $\leq_{3-t t^{-}}$-complete) that is not $\leq_{1-t t}^{\mathrm{P}}$-complete.

2. If for some $\epsilon>0, \mathrm{NP} \cap c o-\mathrm{NP}$ contains a DTIME $\left(2^{n^{\epsilon}}\right)$-bi-immune set, then $\mathrm{NP}$ contains a set that is $\leq_{2-t t^{-}}^{\mathrm{P}}$ complete that is not $\leq_{1-t t^{-}}^{\mathrm{P}}$ complete.

3. If for some $\epsilon>0, \mathrm{UP} \cap c o$-UP contains a DTIME $\left(2^{n^{\epsilon}}\right)$-bi-immune set, then $\mathrm{NP}$ contains a set that is $\leq_{T}^{\mathrm{P}}$-complete that is not $\leq_{t t}^{\mathrm{P}}$-complete.

\section{Preliminaries}

We use standard notation for polynomial-time reductions [LLS75] and we assume that readers are familiar with Turing, $\leq_{T}^{\mathrm{P}}$, and many-one, $\leq_{m}^{\mathrm{P}}$, reducibili- 
ties. Given any positive integer $k>0$, a $k$-Turing reduction $\left(\leq_{k-T}^{P}\right)$ is a Turing reduction that on each input word makes at most $k$ queries to the oracle. A set $A$ is truth-table reducible to a set $B\left(A \leq_{t t}^{\mathrm{P}} B\right)$ if there exist polynomial-time computable functions $g$ and $h$ such that on input $x, g(x)$, for some $m \geq 0$, is (an encoding of) a set of queries $Q=\left\{q_{1}, q_{2}, \cdots, q_{m}\right\}$, and $x \in A$ if and only if $h\left(x, B\left(q_{1}\right), \cdots, B\left(q_{m}\right)\right)=1$. For a constant $k>0, A$ is $k$-truth-table reducible to $B\left(A \leq_{k-t t}^{P} B\right)$ if for all $x,\|Q\|=k$. Given a polynomial-time reducibility $\leq_{r}^{\mathrm{P}}$, recall that a set $S$ is $\leq_{r}^{\mathrm{P}}$-complete for NP if $S \in \mathrm{NP}$ and every set in NP is $\leq_{r}^{\mathrm{P}}$-reducible to $S$.

A language is $\operatorname{DTIME}(T(n))$-complex if $L$ does not belong to $\operatorname{DTIME}(T(n))$ almost everywhere; that is, every Turing machine $M$ that accepts $L$ runs in time greater than $T(|x|)$, for all but finitely many words $x$. A language $L$ is immune to a complexity class $\mathcal{C}$, or $\mathcal{C}$-immune, if $L$ is infinite and no infinite subset of $L$ belongs to $\mathcal{C}$. A language $L$ is bi-immune to a complexity class $\mathcal{C}$, or $\mathcal{C}$-biimmune, if both $L$ and $\bar{L}$ are $\mathcal{C}$-immune. Balcázar and Schöning [BS85] proved that for every time-constructible function $T, L$ is $\operatorname{DTIME}(T(n))$-complex if and only if $L$ is bi-immune to $\operatorname{DTIME}(T(n))$. We will use the following property of bi-immune sets. See Balcázar et al. [BDG90] for a proof.

Proposition 1. Let $L$ be a $\operatorname{DTIME}(T(n))$-bi-immune language and $A$ be an infinite set in $\operatorname{DTIME}(T(n))$. Then both $A \cap L$ and $A \cap \bar{L}$ are infinite.

\section{Separation Results}

Our first goal is to separate $\leq_{2-T}^{\mathrm{P}}$-completeness from $\leq_{m}^{\mathrm{P}}$-completeness under the assumption that NP contains a $\operatorname{DTIME}\left(2^{2 n}\right)$-bi-immune language.

Theorem 1. If $\mathrm{NP}$ contains a $\mathrm{DTIME}\left(2^{2 n}\right)$-bi-immune language, then $\mathrm{NP}$ contains a $\leq_{2-T}^{\mathrm{P}}$-complete set $S$ that is not $\leq_{m}^{\mathrm{P}}$-complete.

Proof. Let $L$ be a DTIME $\left(2^{2 n}\right)$-bi-immune language in NP. Let $k>0$ be a positive integer such that $L \in \operatorname{DTIME}\left(2^{n^{k}}\right)$. Let $M$ decide $L$ in $2^{n^{k}}$ time. Define

$$
\begin{aligned}
t_{1} & =2^{k}, \text { and, for } i \geq 1, \\
t_{i+1} & =\left(t_{i}\right)^{k^{2}},
\end{aligned}
$$

and, for each $i \geq 1$, define

$$
I_{i}=\left\{x\left|t_{i}^{1 / k} \leq\right| x \mid<t_{i}^{k}\right\} .
$$

Observe that $\left\{I_{i}\right\}_{i \geq 1}$ partitions $\Sigma^{*}-\{x|| x \mid<2\}$. Define the following sets:

$$
\begin{aligned}
E & =\cup_{i} \text { even } I_{i}, \\
O & =\cup_{i} \text { odd } I_{i}, \\
L_{e} & =L \cap E, \\
L_{o} & =L \cap O, \\
\text { PadSAT } & =\operatorname{SAT} \cap E .
\end{aligned}
$$


Since $L$ belongs to NP, $L_{e}$ and $L_{o}$ also belong to NP. We can easily see that PadSAT is NP-complete.

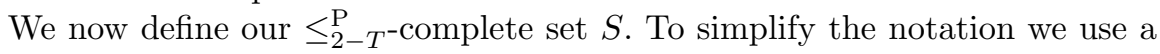
three letter alphabet.

$$
S=0\left(L_{e} \cup \operatorname{PadSAT}\right) \cup 1\left(L_{e} \cap \operatorname{PadSAT}\right) \cup 2 L_{e} .
$$

It is easy to see that $S$ is $\leq_{2-T^{-}}^{\mathrm{P}}$-complete: To determine whether a string $x$ belongs to PadSAT, first query whether $x \in L_{e}$. If $x \in L_{e}$, then $x \in$ PadSAT if and only if $x \in\left(L_{e} \cap\right.$ PadSAT $)$, and, if $x \notin L_{e}$, then $x \in$ PadSAT if and only if $x \in\left(L_{e} \cup \operatorname{PadSAT}\right)$. The same reduction, since it consists of three distinct queries, demonstrates also that $S$ is $\leq_{3-t t}^{\mathrm{P}}$-complete for NP.

The rest of the proof is to show that $S$ is not $\leq_{m}^{\mathrm{P}}$-complete for NP. So assume otherwise and let $f$ be a polynomial-time computable many-one reduction of $L_{o}$ to $S$. We will show this contradicts the hypothesis that $L$ is $\operatorname{DTIME}\left(2^{2 n}\right)$-biimmune.

We need the following lemmas about $L_{o}$. Note that $L_{o} \subseteq O$.

Lemma 1. Let $A$ be an infinite subset of $O$ that can be decided in $2^{2 n}$ time. Then both the sets $A \cap L_{o}$ and $A \cap \overline{L_{o}}$ are infinite.

Proof. Since $A$ is a subset of $O$, a string $x$ in $A$ belongs to $L_{o}$ if and only if it belongs to $L$. Thus $A \cap L_{o}$ is infinite if and only if $A \cap L$ is infinite. Similarly, $A \cap \overline{L_{o}}$ is infinite if and only if $A \cap \bar{L}$ is infinite. Since $A$ can be decided in $2^{2 n}$ time, and $L$ is $2^{2 n}$-bi-immune, by Proposition 1, both the sets $A \cap L$ and $A \cap \bar{L}$ are infinite. Thus, $A \cap L_{o}$ and $A \cap \overline{L_{o}}$ are infinite.

Lemma 2. Let $A$ belong to $\operatorname{DTIME}\left(2^{n^{k}}\right)$, and suppose that $g$ is $a \leq_{m}^{\mathrm{P}}$-reduction from $L_{o}$ to $A$. Then the set

$$
T=\left\{\left.x \in O|| g(x)|<| x\right|^{1 / k}\right\}
$$

is finite.

Proof. It is clear that $T \in \mathrm{P}$. Recall that $M$ is a deterministic algorithm that correctly decides $L$. Let $N$ decide $A$ in $2^{n^{k}}$ time. The following algorithm correctly decides $L$ and runs in $2^{n}$ time on all strings belonging to $T$ : On input $x$, if $x$ does not belong to $T$, then run $M$ on $x$. If $x \in T$, then $x \in L$ if and only if $x \in L_{o}$, so run $N$ on $g(x)$ and accept if and only if $N$ accepts $g(x) . N$ takes $2^{|g(x)|^{k}}$ steps on $g(x)$. Since $|g(x)|<|x|^{1 / k}, N$ runs in $2^{|x|}$ time. Thus, the algorithm runs in $2^{n}$ steps on all strings belonging to $T$. Unless $T$ is finite, this contradicts the fact that $L$ is $\operatorname{DTIME}\left(2^{2 n}\right)$-bi-immune.

Next we show that the reduction should map almost all the strings of $O$ to strings of form by, where $y \in E$ and $b \in\{0,1,2\}$.

Lemma 3. Let

$$
A=\{x \mid x \in O, f(x)=b y, \text { and } y \in O\} .
$$

Then $A$ is finite. 
Proof. It is easy to see that $A$ belongs to P. Both PadSAT and $L_{e}$ are subsets of $E$. Thus if a string $b y$ belongs to $S$, where $b \in\{0,1,2\}$, then $y \in E$. For every string $x$ in $A, f(x)=b y$ and $y \in O$. Thus by $\notin S$, which implies, since $f$ is a many-one reduction from $L_{o}$ to $S$, that $x \notin L_{o}$. Thus $A \cap L_{o}$ is empty. Since $A \subseteq O$, if $A$ were infinite, then this would contradict Lemma 1 , so $A$ is finite.

Thus, for all but finitely many $x$, if $x \in O$ and $f(x)=b y$, then $y \in E$. Now we consider the following set $B$,

$$
B=\left\{x|| x \mid=t_{i} \text { and } i \text { is odd }\right\} .
$$

Observe that $B \in \mathrm{P}$ and that $B$ is an infinite subset of $O$. Thus, by Lemma 1, $B \cap L_{o}$ is an infinite set. Since, for all strings $x, x \in L_{o} \Leftrightarrow f(x) \in S$, it follows that $f$ maps infinitely many of the strings in $B$ into $S$. The rest of the proof is dedicated to showing a contradiction to this fact. Exactly, we define the sets

$$
\begin{aligned}
& B_{0}=\{x \in B \mid f(x)=0 y\}, \\
& B_{1}=\{x \in B \mid f(x)=1 y\}, \text { and } \\
& B_{2}=\{x \in B \mid f(x)=2 y\},
\end{aligned}
$$

and we prove that each of these sets is finite.

Lemma 4. $B_{0}$ is finite.

Proof. Assume $B_{0}$ is infinite. Let

$$
C=\left\{x \in B_{0} \mid f(x)=0 y \text { and } y \in E\right\} .
$$

Since $B_{0}$ is a subset of $O$, by Lemma 3 , for all but finitely strings in $B_{0}$, if $f(x)=0 y$, then $y \in E$. Thus $B_{0}$ is infinite if and only if $C$ is infinite.

Consider the following partition of $C$.

$$
\begin{aligned}
& C_{1}=\left\{\left.x \in C|f(x)=0 y,| y|<| x\right|^{1 / k}\right\}, \\
& C_{2}=\left\{\left.x \in C|f(x)=0 y,| x\right|^{1 / k} \leq|y|<|x|^{k}\right\}, \\
& C_{3}=\left\{\left.x \in C|f(x)=0 y,| y|\geq| x\right|^{k}\right\} .
\end{aligned}
$$

We will show that each of the sets $C_{1}, C_{2}$, and $C_{3}$ is finite.

Claim $1 C_{1}$ is finite.

Proof. Since $S \in \operatorname{DTIME}\left(2^{n^{k}}\right)$, the claim follows from Lemma 2 .

Claim $2 C_{2}$ is the empty set.

Proof. Assume that $x \in C_{2}$. Since $C_{2} \subseteq C \subseteq B,|x|=t_{i}$, for some odd $i$. So, $|x|^{1 / k} \leq|y|<|x|^{k}$ implies that $t_{i}^{1 / k} \leq|y|<t_{i}^{k}$, which implies $y \in I_{i}$. Since $i$ is odd, $y \in O$. However, by definition of $C, y \in E$. Thus, $C_{2}=\emptyset$. 
Claim $3 C_{3}$ is finite.

Proof. Observe that $C_{3} \in \mathrm{P}$. Suppose $C_{3}$ is infinite. Define $C_{4}=C_{3}-L_{o}$. We first show, under the assumption $C_{3}$ is infinite, that $C_{4}$ is infinite. Suppose $C_{4}$ is finite. Then the set $C_{5}=C_{3} \cap L_{o}$ differs from $C_{3}$ by a finite set. Thus, since $C_{3} \in \mathrm{P}, C_{5} \in \mathrm{P}$ also. At this point, we know that $C_{5}$ is an infinite subset of $O$ that belongs to $\mathrm{P}$, and that $C_{5}$ is a subset of $L_{o}$. Thus, $C_{5} \cap \overline{L_{o}}$ is empty, which contradicts Lemma 1. Thus, $C_{4}$ is an infinite subset of $C_{3}$.

Let

$$
F=\left\{y \in E \mid \exists x\left[x \in O, x \notin L_{o}, f(x)=0 y \text {, and }|y| \geq|x|^{k}\right]\right\} .
$$

The following implications show that $F$ is infinite:

$$
\begin{gathered}
C_{4} \text { is infinite } \\
\Rightarrow \quad \\
\exists^{\infty} x\left[x \in O, x \notin L_{o}, f(x)=0 y,|y| \geq|x|^{k}, y \in E\right] \\
\Rightarrow \\
\exists^{\infty} y \in E\left[\exists x x \in O, x \notin L_{o}, f(x)=0 y,|y| \geq|x|^{k}\right] .
\end{gathered}
$$

For each string $y$ in $F$, there exists a string $x \in O-L_{o}$ such that $f(x)=0 y$. Since $f$ is a many-one reduction from $L_{o}$ to $S, f(x)=0 y \notin S$. Thus $y \notin$ $L_{e} \cup \operatorname{PadSAT}$, and so $y \notin L_{e}$. However, since $y \in E$, we conclude that $y \notin L$. Thus, $F$ is an infinite subset of $\bar{L}$.

Now we contradict the fact that $L$ is $\operatorname{DTIME}\left(2^{2 n}\right)$-bi-immune by showing that $F$ is decidable in time $2^{2 n}$. Let $y$ be an input string. First decide, in polynomial time, whether $y$ belongs to $E$. If $y \notin E$, then $y \notin F$. If $y \in E$, compute the set of all $x$ such that $|x| \leq|y|^{1 / k}, x \in O$, and $f(x)=0 y$. Run $M$ on every string $x$ in this set until $M$ rejects one of them. Since $x \in O, M$ rejects a string $x$ only if $x \notin L_{o}$. If such a string is found, then $y \in F$, and otherwise $y \notin F$. There are at most $2 \times 2^{|y|^{1 / k}}$ many $x$ 's such that $|x| \leq|y|^{1 / k}$ and $f(x)=0 y$. The time taken to run $M$ on each such $x$ is at most $2^{|x|^{k}} \leq 2^{|y|}$. Thus, the total time to decide whether $y \in F$ is at most $2^{|y|} \times 2^{|y|^{1 / k}} \times 2 \leq 2^{2|y|}$. Thus, $F$ is decidable in time $2^{2 n}$.

We conclude that $F$ must be a finite set. Therefore, $C_{4}$ is finite, from which it follows that $C_{3}$ is finite.

Each of the claims is established. Thus, $C=C_{1} \cup C_{2} \cup C_{3}$ is a finite set, and this proves that $B_{0}$ is a finite set.

Lemma 5. $B_{1}$ is a finite set.

Proof. Much of the proof is similar to the proof of Lemma 4. Assume that $B_{1}$ is infinite. This time, define

$$
C=\left\{x \in B_{1} \mid f(x)=1 y \text { and } y \in E\right\} .
$$


By Lemma 3, $C$ is infinite if and only if $B_{1}$ is infinite. Thus, by our assumption, $C$ is infinite. Partition $C$ as follows.

$$
\begin{aligned}
& C_{1}=\left\{\left.x \in C|f(x)=1 y,| y|<| x\right|^{1 / k}\right\} \\
& C_{2}=\left\{\left.x \in C|f(x)=1 y,| x\right|^{1 / k} \leq|y|<|x|^{k}\right\} \\
& C_{3}=\left\{\left.x \in C|f(x)=1 y,| y|\geq| x\right|^{k}\right\}
\end{aligned}
$$

As in the proof of Lemma 4 , we can show that $C_{1}$ is a finite set and $C_{2}$ is empty. Now we proceed to show that $C_{3}$ is also a finite set.

Claim $4 C_{3}$ is finite.

Proof. Assume $C_{3}$ is infinite and observe that $C_{3} \in \mathrm{P}$. Define $C_{4}=C_{3} \cap L_{o}$. Now we show that $C_{4}$ is infinite. If $C_{4}$ is finite, then $C_{5}=C_{3}-L_{o}$ contains all but finitely many strings of $C_{3}$. Thus, since $C_{3}$ belongs to $\mathrm{P}, C_{5}$ also belongs to $\mathrm{P}$. Thus $C_{5}$ is an infinite subset of $O$ that belongs to $\mathrm{P}$, for which $C_{5} \cap L_{o}$ is empty. That contradicts Lemma 1 . Thus, $C_{4}$ is infinite.

Consider the following set:

$$
F=\left\{y \in E \mid \exists x\left[x \in L_{o}, f(x)=1 y,|y| \geq|x|^{k}\right]\right\}
$$

The following implications show that $F$ is infinite.

$$
\begin{gathered}
C_{4} \text { is infinite } \\
\Rightarrow \\
\exists^{\infty} x\left[x \in L_{o}, f(x)=1 y,|y| \geq|x|^{k}, y \in E\right] \\
\Rightarrow \\
\exists^{\infty} y\left[\exists x f(x)=1 y,|y| \geq|x|^{k}, x \in L_{o}, y \in E\right] .
\end{gathered}
$$

For each string $y \in F$, there exists a string $x \in L_{o}$ such that $f(x)=1 y$. Since $f$ is a $\leq_{m}^{\mathrm{P}}$-reduction from $L_{o}$ to $S, f(x)=1 y \in S$, so $y \in L_{e} \cap$ PadSAT. In particular, $y \in L_{e} \subseteq L$. Therefore, $F$ is an infinite subset of $L$. However, as in the proof of Claim 3, we can decide whether $y \in F$ in $2^{2|y|}$ steps, which contradicts the fact that $L$ is $\operatorname{DTIME}\left(2^{2 n}\right)$-bi-immune: Let $y$ be an input string. First decide whether $y \in E$, and if not, then reject. If $y \in E$, then search all strings $x$ such that $|x| \leq|y|^{1 / k}, x \in O$, and $f(x)=1 y$. For each such $x$, run $M$ on $x$ to determine whether $x \in L \cap O=L_{o}$. If an $x \in L_{o}$ is found, then $y \in F$, and otherwise $y \notin F$. The proof that this algorithm runs in $2^{2 n}$ steps is identical to the argument in the proof of Claim 3.

Therefore, $F$ is finite, from which it follows that $C_{4}$ is finite, and so $C_{3}$ must be finite.

Now we know that $C$ is finite. This proves that $B_{1}$ is finite, which completes the proof of Lemma 5 .

Lemma 6. $B_{2}$ is a finite set. 
Proof. Assume $B_{2}$ is infinite. Then

$$
C=\{x \in B \mid f(x)=2 y, \text { and } y \in E\}
$$

is infinite. We partition $C$ into

$$
\begin{aligned}
& C_{1}=\left\{\left.x \in C|f(x)=2 y,| y|<| x\right|^{1 / k}\right\} \\
& C_{2}=\left\{\left.x \in C|f(x)=2 y,| x\right|^{1 / k} \leq|y|<|x|^{k}\right\} \\
& C_{3}=\left\{\left.x \in C|f(x)=2 y,| y|\geq| x\right|^{k}\right\}
\end{aligned}
$$

The proofs that $C_{1}, C_{2}$, and $C_{3}$ are finite are identical to the arguments in the proof of Lemma 5. (In particular, it suffices to define $F$ as in the proof of Lemma 5.)

Now we have achieved our contradiction, for we have shown that the each of the sets $B_{1}, B_{2}$, and $B_{3}$ are finite. Therefore, $f$ cannot map infinitely many of the strings in $B$ into $S$, which proves that $f$ cannot be a $\leq_{m}^{\mathrm{P}}$-reduction from $L_{o}$ to $S$. Therefore, $S$ is not $\leq_{m}^{\mathrm{P}}$-complete.

Next we show that NP has a $\operatorname{DTIME}\left(2^{n^{\epsilon}}\right)$-bi-immune set if and only if NP has a DTIME $\left(2^{n^{k}}\right)$-bi-immune set using a reverse padding trick [ASTZ97].

Theorem 2. Let $0<\epsilon<1$ and $k$ be any positive integer. NP has a $\operatorname{DTIME}\left(2^{n^{\epsilon}}\right)$ bi-immune set if and only if $\mathrm{NP}$ has a $\operatorname{DTIME}\left(2^{n^{k}}\right)$-bi-immune set.

Proof. The implication from right to left is obvious. Let $L \in \operatorname{NP}$ be a $\operatorname{DTIME}\left(2^{n^{\epsilon}}\right)$ bi-immune set. Define

$$
L^{\prime}=\left\{x\left|0^{n^{k / \epsilon}} x \in L,\right| x \mid=n\right\}
$$

and observe that $L^{\prime} \in \mathrm{NP}$. We claim that $L^{\prime}$ is DTIME $\left(2^{n^{k}}\right)$-bi-immune. Suppose otherwise. Then there exists an algorithm $M$ that decides $L^{\prime}$ and $M$ runs in $2^{n^{k}}$ steps on infinitely many strings. Consider the following algorithm for $L$ :

input $y$;

if $y=0^{n^{k / \epsilon}} x(|x|=n)$

then $\operatorname{run} M$ on $x$

and accept $y$ if and only if $M$ accepts $x$

else run a machine that decides $L$;

Since $M$ runs in $2^{n^{k}}$ time on infinitely many $x$, the above algorithm runs in time $2^{|x|^{k}}$ steps on infinitely many strings of the form $y=0^{|x|^{k / \epsilon}} x$. Observe that $|y| \geq|x|^{n^{k / \epsilon}}$. Thus, the above algorithm runs in $2^{|y|^{\epsilon}}$ steps on infinitely many $y$. This contradicts the $\operatorname{DTIME}\left(2^{n^{\epsilon}}\right)$-bi-immunity of $L$.

Corollary 1. If NP contains a $2^{n^{\epsilon}}$-bi-immune language, then NP contains a $\leq_{2-T}^{\mathrm{P}}$-complete set $S$ that is not $\leq_{m}^{\mathrm{P}}$-complete. 
The proof of the next theorem shows that we can extend the proof of The-

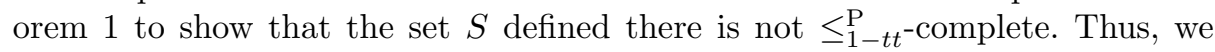
arrive at our main result.

Theorem 3. If NP contains a $2^{n^{\epsilon}}$-bi-immune language, then NP contains a $\leq_{2-T}^{\mathrm{P}}$-complete set $S$ that is not $\leq_{1-t t}^{\mathrm{P}}$-complete.

Proof. The proof is a variation of the proof of Theorem 1, and we demonstrate the interesting case only. Assume that the set $S$ defined there is $\leq_{1-t t}^{\mathrm{P}}$-complete and let $(g, h)$ be a 1-truth-table reduction from $L_{o}$ to $S$. Recall that, for each string $x, g(x)$ is a query to $S$ and that

$$
x \in L_{o} \Leftrightarrow h(x, S(g(x)))=1 .
$$

The function $h$ on input $x$ implicitly defines four possible truth-tables. Let us define the sets

$$
\begin{aligned}
& T=\{x \mid h(x, 1)=1 \text { and } h(x, 0)=1\}, \\
& F=\{x \mid h(x, 1)=0 \text { and } h(x, 0)=0\}, \\
& Y=\{x \mid h(x, 1)=1 \text { and } h(x, 0)=0\}, \\
& N=\{x \mid h(x, 1)=0 \text { and } h(x, 0)=1\} .
\end{aligned}
$$

Each of the sets $T, F, Y$, and $N$ belongs to P. Also, $T \subseteq L_{o}, F \subseteq \overline{L_{o}}$, for all strings $x \in Y$,

$$
x \in L_{o} \Leftrightarrow x \in S,
$$

and for all strings $x \in N$,

$$
x \in L_{o} \Leftrightarrow x \in \bar{S} .
$$

It follows immediately that $T$ and $F$ are finite sets. Now, as we did in the proof of Theorem 1, we consider the set $B=\left\{x|| x \mid=t_{i}\right.$ and $i$ is odd $\}$. Recall that $B \in \mathrm{P}$ and that $B$ is an infinite subset of $O$. For all but finitely many strings $x \in B$, either $x \in Y$ or $x \in N$. In order to illustrate the interesting case, let us assume that $B^{N}=B \cap N$ is infinite. Note that $B^{N} \in \mathrm{P}$ and that $B^{N}$ is an infinite subset of $O$. By Lemma $1, B^{N} \cap \overline{L_{o}}$ is infinite. For all $x \in B^{N}$, $x \in \overline{L_{o}} \Leftrightarrow x \in S$. Thus, $g$ maps infinitely many of the strings in $B^{N}$ into $S$. Similar to our earlier analysis, we contradict this by showing that each of the following sets is finite:

$$
\begin{aligned}
& B_{0}=\left\{x \in B^{N} \mid g(x)=0 y\right\}, \\
& B_{1}=\left\{x \in B^{N} \mid g(x)=1 y\right\}, \\
& B_{2}=\left\{x \in B^{N} \mid g(x)=2 y\right\} .
\end{aligned}
$$

Here we will demonstrate that $B_{0}$ is finite. The other cases will follow similarly.

Define $A=\left\{x \in B_{0} \mid g(x)=b y\right.$, and $\left.y \in O\right\}$. Again we need to show that $A$ is a finite set, but we need a slightly different proof from that for Lemma 3. Note 
that $A \in$ P. If $g(x)=0 y \in S$, then $y \in E$. Thus, $x \in A \Rightarrow g(x) \notin S \Rightarrow x \in L_{o}$. Thus $A \subseteq L_{o}$, from which it follows that $A$ is finite. Hence, the set

$$
C=\left\{x \in B_{0} \mid g(x)=0 y \text { and } y \in E\right\}
$$

is an infinite set. As earlier, we partition $C$ into the sets

$$
\begin{aligned}
& C_{1}=\left\{\left.x \in C|f(x)=0 y,| y|<| x\right|^{1 / k}\right\}, \\
& C_{2}=\left\{\left.x \in C|f(x)=0 y,| x\right|^{1 / k} \leq|y|<|x|^{k}\right\}, \\
& C_{3}=\left\{\left.x \in C|f(x)=0 y,| y|\geq| x\right|^{k}\right\},
\end{aligned}
$$

and we show that each of these sets is finite. To show that $C_{1}$ is finite, we show more generally, as in the proof of Lemma 2, that $V=\left\{\left.x \in B^{N}|| g(x)|<| x\right|^{1 / k}\right\}$ is a finite set. (The critical fact is that for $x \in V, x \in S \Leftrightarrow x \in \overline{L_{o}} \Leftrightarrow x \notin L$, because $V \subseteq O$.) Also, it is easy to see that $C_{2}=\emptyset$.

We need to show that $C_{3}$ is finite. Assume that $C_{3}$ is infinite. Noting that $C_{3} \in \mathrm{P}$, the proof of Claim 4 (not Claim 3!) shows that the set $C_{4}=C_{3} \cap L_{o}$ is infinite. Then,

$$
\begin{gathered}
\exists^{\infty} x\left[x \in C_{4}, g(x)=0 y,|y|<|x|^{1 / k}\right] \\
\Rightarrow \\
\exists^{\infty} x\left[x \in B^{N}, x \in L_{o}, y \in E, g(x)=0 y,|y|<|x|^{1 / k}\right] \\
\Rightarrow \\
\exists^{\infty} y \exists x\left[x \in B^{N}, x \in L_{o}, y \in E, g(x)=0 y,|y|<|x|^{1 / k}\right] .
\end{gathered}
$$

Thus, the set

$$
U=\left\{y \mid \exists x\left[x \in B^{N}, x \in L_{o}, y \in E, g(x)=0 y,|y|<|x|^{1 / k}\right]\right\}
$$

is infinite. For each string $y \in U$, there exists $x \in B^{N} \cap L_{o}$ such that $g(x)=0 y$. For each such $x, g(x)=0 y \in \bar{S}$. Thus, $y \notin L_{e} \cup \operatorname{PadSAT}$, so, in particular, $y \notin L_{e}$. However, $y \in E$, so $y \in L$. Thus, $U$ is an infinite subset of $\bar{L}$.

Now we know that $C$ is finite, from which it follows that $B_{0}$ is a finite set. In a similar manner we can prove that $B_{1}$ and $B_{2}$ are finite, which completes the proof of the case that $B^{N}$ is infinite. The other possibility, that $B^{Y}=B \cap Y$ is infinite can be handled similarly.

There is no previous work that indicates a separation of $\leq_{2-t t}^{\mathrm{P}}$-completeness

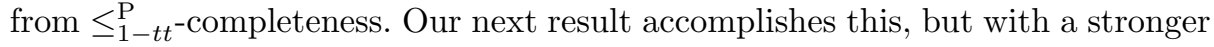
hypothesis.

Theorem 4. If $\mathrm{NP} \cap$ co-NP contains a $2^{n^{\epsilon}}$-bi-immune set, then NP contains a $\leq_{2-t t}^{\mathrm{P}}$-complete set that is not $\leq_{1-t t}^{\mathrm{P}}$-complete.

Proof. The hypothesis implies the existence of a $2^{n^{k}}$-bi-immune language $L$ in $\mathrm{NP} \cap$ co-NP. Let

$$
S=0\left(L_{e} \cap \operatorname{PadSAT}\right) \cup 1\left(\left(E-L_{e}\right) \cap \operatorname{PadSAT}\right) .
$$


Since $L$ belongs to NP $\cap$ co-NP, $S$ belongs to NP. Since both PadSAT and $L_{e}$ are subsets of $E$, for any string $x$

$$
x \in \operatorname{PadSAT} \Leftrightarrow\left(x \in L_{e} \cap \operatorname{PadSAT}\right) \vee\left(x \in\left(E-L_{e}\right) \cap \mathrm{PadSAT}\right) .
$$

Thus $S$ is 2-tt-complete for NP. The rest of the proof is similar to the proof of Theorem 3.

\section{Acknowledgements}

The authors benefitted from conversations about this work with Osamu Watanabe, and with Lance Fortnow and Jack Lutz.

\section{References}

[ASB00] K. Ambos-Spies and L. Bentzien. Separating NP-completeness under strong hypotheses. Journal of Computer and System Sciences, 61(3):335-361, 2000.

[ASTZ97] K. Ambos-Spies, A. Terwijn, and X. Zheng. Resource bounded randomness and weakly complete problems. Theoretical Computer Science, 172(1):195207, 1997.

[BDG90] J. Balcázar, J. Diaz, and J. Gabarró. Structural Complexity II. SpringerVerlag, Berlin, 1990.

[BS85] J. Balcázar and U. Schöning. Bi-immune sets for complexity classes. Mathematical Systems Theory, 18(1):1-18, June 1985.

[JK76] D. Johnson and S. Kashdan. Lower bounds for selection in $x+y$ and other multisets. Technical Report 183, Pennsylvania State Univ., University Park, PA, 1976.

[LLS75] R. Ladner, N. Lynch, and A. Selman. A comparison of polynomial time reducibilities. Theoretical Computer Science, 1:103-123, 1975.

[LM96] J. Lutz and E. Mayordomo. Cook versus Karp-Levin: Separating completeness notions if NP is not small. Theoretical Computer Science, 164:141-163, 1996.

[LY90] L. Longpré and P. Young. Cook reducibility is faster than Karp reducibility. Journal of Computer and System Sciences, 41:389-401, 1990.

[PS01] A. Pavan and A. Selman. Separation of NP-completeness notions. In 16th Annual IEEE Conference on Computational Complexity, pages 78-89, 2001.

[Sel82] A. Selman. Reductions on NP and P-selective sets. Theoretical Computer Science, 19:287-304, 1982.

[SG77] I. Simon and J. Gill. Polynomial reducibilities and upward diagonalizations. Proceedings of the Ninth Annual ACM Symposium on Theory of Computing, pages 186-194, 1977. 